\title{
Scientific publishing in the COVID-19 era: successes and pitfalls
}

\section{Antonio Federico ${ }^{1}$}

Published online: 2 July 2020

(C) Fondazione Società Italiana di Neurologia 2020

The interest in neurologic research concerning the COVID pandemic continues to be very high as illustrated by the enormous increase in the number of articles published or present online in the most prestigious scientific journals, from The Lancet, with more than 600 articles since April, to The New England Journal of Medicine, with more than 190, to the neurologic journals such as European Neurology (32), the Journal of Neurolology (20), the Journal of Neurological Sciences (16), and Neurological Sciences (21). When we checked Orphanet, on 20 June 2020, there were more than 23,000 articles related to COVID-19.

Table 1 shows the influx of articles to our Journal, Neurological Sciences, in the past 3 months.

All of the journals organized an expediated article evaluation, to enable the scientific community to share, as soon as possible, the emerging information regarding mitigating the effects of the pandemic on human health and providing suggestions concerning drug efficacy and the development of new care organizations.

The recent retraction of two articles by several authors has put focus on the problem of accuracy regarding big data control and the risks of an accelerated, possibly less accurate, publication system.

In fact, two studies of drug therapy and COVID-19 have been retracted from two different prestigious journals regarding the quality of the data obtained from an international database held by Surgisphere Corporation, which included electronic health records from 169 hospitals on three continents and was not controlled by the authors.

The Lancet retracted the article titled "Hydroxychloroquine or Chloroquine With or Without a Macrolide for Treatment of COVID-19: A Multinational Registry Analysis" [1].

Antonio Federico

federico@unisi.it

1 Department of Medicine, Surgery and Neurosciences, Medical School, University of Siena, Viale Bracci 2, 53100 Siena, Italy
The New England Journal of Medicine retracted the paper titled "Cardiovascular Disease, Drug Therapy, and Mortality in COVID-19" [2]. The study examined the effect of preexisting treatment with angiotensin-converting enzyme (ACE) inhibitors and angiotensin-receptor blockers (ARBs) on COVID-19.

In their retraction letter to The Lancet, the authors noted that an independent review of the data was not possible because Surgisphere Corporation, which holds the database, "would not transfer the full dataset, client contracts, and the full ISO audit report to their servers for analysis as such transfer would violate client agreements and confidentiality requirements ... Based on this development, we can no longer vouch for the veracity of the primary data sources."

In their retraction letter to The New England Journal of Medicine, the authors wrote: "Because all the authors were not granted access to the raw data and the raw data could not be made available to a third-party auditor, we are unable to validate the primary data sources underlying our article."

The high volume of scientific data necessitates a thorough control not only by the editorial staff of the journals and reviewer for the quality of the information but also, and most of all, by the authors.

The "Publish or Perish" statement has been recently considered as a guideline for the publication system and career development. The thorough evaluation of the papers by independent reviewers, frequently available with difficulty by Journal Editors, has been considered a valid system to select the best articles and to confirm the value of the research, although many debates on the topic have occurred in the past years. The COVID-19 pandemic has resulted in an extraordinary output of research regarding the novel pathological condition involving many millions of patients; however, this has necessitated an accelerated system of evaluation before publication, which was not easy to organize and that has sometimes failed. This unprecedented increase in articles related to COVID-19, more than 23,000 from January to June, necessitates serious consideration regarding the methods used to guarantee the quality of the scientific results published and 
Table 1 COVID-19

related articles and

Neurological Sciences

from 15 March to 20

June 2020

\begin{tabular}{ll}
\hline Articles received & 85 \\
Articles accepted & 39 \\
Articles rejected & 27 \\
Articles under evaluation & 19 \\
\hline
\end{tabular}

to avoid a reduction in the data quality. Many aspects effect this outcome, such as the short time to evaluate the papers, the fast online publication of the results even before the reviewers' evaluation, and the frequent use of the results by the media that often emphasize the reported data without scientific criticism. Self-control of the science process, based on ethical rules and the knowledge of the possible practical consequences of low level and incorrect scientific information, needs to be the guideline in all publication processes [3]. This self-control needs to be practiced by all people involved in the research system, such as the authors, the reviewers, the editorial boards, the stakeholders, the supporting research agencies, the universities, and all the research institutions, in the interest of the quality of science and the practical utilization of the results that must be protected and preserved.

\section{COVID-19 and Neurological Sciences: new articles in the July issue}

This issue presents new articles related to the COVID-19 pandemic and neurology.

\section{Control of neurologic diseases by remote monitoring technologies}

Dalla Costa et al. [4], in a research coordinated by Prof. Comi, described the real-time assessment of COVID-19 prevalence and impact among multiple sclerosis patients in a multicenter European study using remote monitoring technologies available through wearable devices and smartphone technology. Their study included 399 RADAR-MS patients (mean age 43.9 years, $60.7 \%$ females) at three designated centers in Milan (Italy), Barcelona (Spain), and Copenhagen (Denmark). Major symptoms suggestive of COVID-19 were reported by $87 / 399$ patients $(21.8 \%, 95 \%$ Cis: $17.8-25.9 \%)$ : $31(7.8 \%)$ had a fever, $73(18.3 \%)$ presented respiratory symptoms, $38(9.5 \%)$ had gastrointestinal disturbances, $17(4.3 \%)$ had conjunctivitis, and 17 (4.3\%) experienced ageusia and anosmia. Two patients had severe COVID-19 manifestations, and no one died. The prevalence of suspected cases varied between 9.3 (95\% Cis: $6.4-12.1$ ) and $13.0 \%$ (95\%Cis: 9.716.3) according to case definitions, and the RT-PCR test for SARS-CoV-2 was performed in only ten patients (four positives). A trend for an increased risk of COVID-19 symptoms under alemtuzumab and cladribine treatments compared to injectables was observed. On an emotional level, $91 \%$ of patients were moderately to deeply worried by the pandemic. The authors conclude that while a minority of MS patients received a laboratory confirmed diagnosis of COVID-19, the prevalence of the infection may have been ten times higher among MS patients across Europe. Remote monitoring technologies may support health authorities in monitoring and containing the ongoing pandemic.

As previously reported by Caso and Federico [5], the epidemic gives the occasion to develop and improve care organization and telemedicine; in particular, Sarti et al. [6] reported on the smart working and telehealth reorganization of a language and learning disorders service in Milan during the COVID-19 pandemic as a model for the assistance and rehabilitation of children with neurodevelopmental disabilities.

\section{Neurologic involvement in SARS-CoV-2 infection}

Fasano et al. [7] reported a case of first motor seizure as a presenting symptom of SARS-CoV-2 infection. The present case further underscores that initial manifestations of novel coronavirus infection might be non-specific, and it highlights the need for physicians to consider potential neurological manifestations of SARS-CoV-2 infection.

Assini et al. [8] presented two new cases of Guillain-Barré syndrome (GBS), with typical onset latency after SARS-Cov2 infection with two very atypical features. In the first case, the impairment of several cranial nerves (I-III-V (motor branch)IX-X-XII) in association with a demyelinating peripheral neuropathy suggests an overlap of Miller Fisher and GBS. In the second case, the massive impairment of the vegetative nervous system, associated with electrophysiological changes typical for acute motor sensory axonal neuropathy (AMSAN), has been emphasized. Although the association between GBS and vegetative symptoms has been previously described, this is the first case of COVID-19-related AMSAN associated with massive vegetative involvement. This case suggests a careful differential diagnosis between AMSAN and critical illness neuropathy. However, the absence of a myogenic pattern on the EMG, the severe vegetative impairment, and the excellent response to immunoglobulins therapy are suggestive of AMSAN diagnosis. Finally, in both cases, the mirror pattern with an increase in Ig both in CSF and serum confirms the autoimmune and inflammatory etiology. Moreover, the absence of COVID-19 in CSF suggests an immuno-mediated inflammatory mechanism.

Manganelli et al. [9] reported three interesting COVID-19 cases where the patients, although they had recovered from pneumonia, could not be weaned from invasive mechanical ventilation. The clinical evaluation was consistent with a brainstem and mainly respiratory center involvement that can explain the weaning failure in patients that were awake 
and had recovered from lung involvement. These data, though limited, indicate that brainstem involvement may play a role in respiratory failure and perhaps in the high death rate of COVID-19 patients. Moreover, the weaning failure from mechanical ventilation due to central respiratory drive depression may underlie the unusually long stay in the ICU reported for COVID-19 patients.

Finally, Niazkar et al. [10] conducted a review article on the neurologic manifestations of COVID-19, confirming the clinical heterogeneity of this syndrome and the possible different neurologic involvements. In addition, Frisullo et al. [11], from the Catholic University of Rome, commenting on the previously reported article by Baracchini et al. [12], described their new stroke integrated care pathway (ICP) during the pandemic. The main novelty of the new stroke ICP is the creation of a sub-intensive ward to treat time-dependent diseases in COVID-19 patients. In this way, a multidisciplinary approach and multiparameter monitoring are guaranteed to a patient presenting as more complex than the patient affected "only" by ischemic stroke, without renouncing the high specialization ensured by the presence of the stroke team. Moreover, this stroke ICP, with a definite separation between the two paths, is the model that best guarantees against the possible contamination of "clean" environments, minimizing risks for non-COVID-19 patients.

\section{Conclusions}

In conclusion, Kwon \& Kim [13], from one of the South Korean hospitals particularly and firstly involved with the COVID-19 infection immediately after China, described their experience with patients affected by COVID-19 and neurologic disorders who received delayed care, determining a worsening clinical evolution. They recommended neurologists to be alert and, as we reported in our previous editorial [5], not be on lockdown.

\section{Compliance with ethical standards}

Conflict of interest The authors declare that they have no conflict of interest.

Ethical approval None required.

\section{References}

1. Mehra MR, Ruschitzka F, Patel AN (2020) Retractionhydroxychloroquine or chloroquine with or without a macrolide for treatment of COVID-19: a multinational registry analysis. Lancet 395(10240):1820. https://doi.org/10.1016/S0140-6736(20) 31324-6

2. Mehra MR, Desai SS, Kuy S, Henry TD, Patel AN (2020) Retraction: cardiovascular disease, drug therapy, and mortality in Covid-19. N Engl J Med. https://doi.org/10.1056/ NEJMoa2007621 N Engl J Med. https://doi.org/10.1056/ NEJMc2021225

3. Shapiro SB, Franco EL (2019) Restoring dignity in academic publishing is a collective duty. JDR Clin Trans Res 4(1):5-8. https:// doi.org/10.1177/2380084418806839

4. Dalla Costa G, Leocani L, Montalban, Guerrero AI, Sorensen PS, Magyari M, Dobson RJB, Cummins N, Narayan VA, Hotopf M, Comi GC (2020) Real-time assessment of COVID-19 prevalence among multiple sclerosis patients: a multicenter European study. Eur Arch Otorhinolaryngol. https://doi.org/10.1007/s00405-02005965-1

5. Caso V, Federico A (2020) No lockdown for neurological diseases during COVID19 pandemic infection. Neurol Sci 41(5):999-1001. https://doi.org/10.1007/s10072-020-04389-3

6. Sarti S, De Salvatore M, Gazzola S, Pantaleoni C, Granocchio E (2020) So far so close: an insight into smart working and telehealth reorganization of a language and learning disorders service in Milan during COVID-19 pandemic. Neurol Sci. https://doi.org/10.1007/ s10072-020-04481-8

7. Fasano A, Cavallieri F, Canali E, Valzania F (2020) First motor seizure as presenting symptom of SARS-CoV-2 infection. Neurol Sci. https://doi.org/10.1007/s10072-020-04460-z

8. Assini A, Benedetti L, Di Maio L, Schirinzi E, Del Sette M (2020) New clinical manifestation of COVID-19 related Guillain-Barrè syndrome highly responsive to intravenous immunoglobulins: two Italian cases. Neurol Sci. https://doi.org/10.1007/s10072-02004484-5

9. Manganelli F, Vargas M, Iovino A, Iacovazzo C, Santoro L, Servillo G (2020) Brainstem involvement and respiratory failure in COVID-19. Neurol Sci. https://doi.org/10.1007/s10072-020$04487-2$

10. Niazkar HR, Zibaee B, Nasimi A, Bahri N (2020) The neurological manifestations of COVID-19: a review article. Neurol Sci. https:// doi.org/10.1007/s10072-020-04486-3

11. Frisullo G, De Belvis AG, Della G, Angioletti C, Calabresi P (2020) Stroke integrated care pathway during COVID-19 pandemic. Neurol Sci. https://doi.org/10.1007/s10072-020-04480-9

12. Baracchini C, Pieroni A, Viaro F, Cianci V, Cattelan AM, Tiberio I, Munari M, Causin F (2020) Acute stroke management pathway during Coronavirus-19 pandemic. Neurol Sci 41(5):1003-1005. https://doi.org/10.1007/s10072-020-04375-9

13. Kwon DH, Kim KT (2020) Keep "time is brain," even in the crisis of COVID-19. Neurol Sci. https://doi.org/10.1007/s10072-02004483-6

Publisher's note Springer Nature remains neutral with regard to jurisdictional claims in published maps and institutional affiliations. 